\title{
Forging a path to a better normal for conferences and collaboration
}

The 2020 COVID-19 pandemic forced a string of cancelled conferences, causing many organizers to shift meetings online, with mixed success. Seizing the opportunity, a group of researchers came together to rethink how the conference experience and collaboration in general can be improved in a more virtual-centric future.

\author{
Vanessa A. Moss, Matt Adcock, Aidan W. Hotan, Rika Kobayashi, Glen A. Rees, Coralie Siégel, \\ Chenoa D. Tremblay and Claire E. Trenham
}

$\mathrm{D}$ oes the future of scientific progress (our conferences, our collaboration, our communication) really rely on a rush back to in-person interaction?

Digital-meeting fatigue, loss of serendipitous hallway conversations, impersonal interactions, challenging time zones: these have been commonly cited as reasons why virtual interaction fundamentally falls short of its in-person counterpart and as justification for a speedy return to 'normal'. But what kind of collaborative future could we, and should we, be aiming for?

There has already been considerable discussion of whether the future of conferences and meetings might be in-person, purely digital, or a hybrid of the two. While some have expressed a desire to return to face-to-face meetings as they were before the COVID-19 pandemic, many seek an altered future for academic collaboration (alternating in-person and virtual, local hubs linked via the internet, or purely virtual have been among popular suggestions). More still sit somewhere in between, empirically aware of the challenges of the virtual format but also acknowledging the benefits gained by collaborating through these new technologies. Parallel to these discussions, a new future of work is already upon us. Many parts of academia and industry are discussing dramatic changes to expectations about the role of the physical office $^{1,2}$ while other parts have essentially, where possible, returned to 'normal'. It is now widely acknowledged that working from home is often feasible and can be more productive, while arguments in favour of the office often focus on the importance of the hallway conversation, sense of community and performance management ${ }^{3}$.

To explore what future interaction might look like, in September 2020 we brought together a cross-disciplinary community for The Future of Meetings (TFOM) symposium (Fig. 1), an event hosted by the

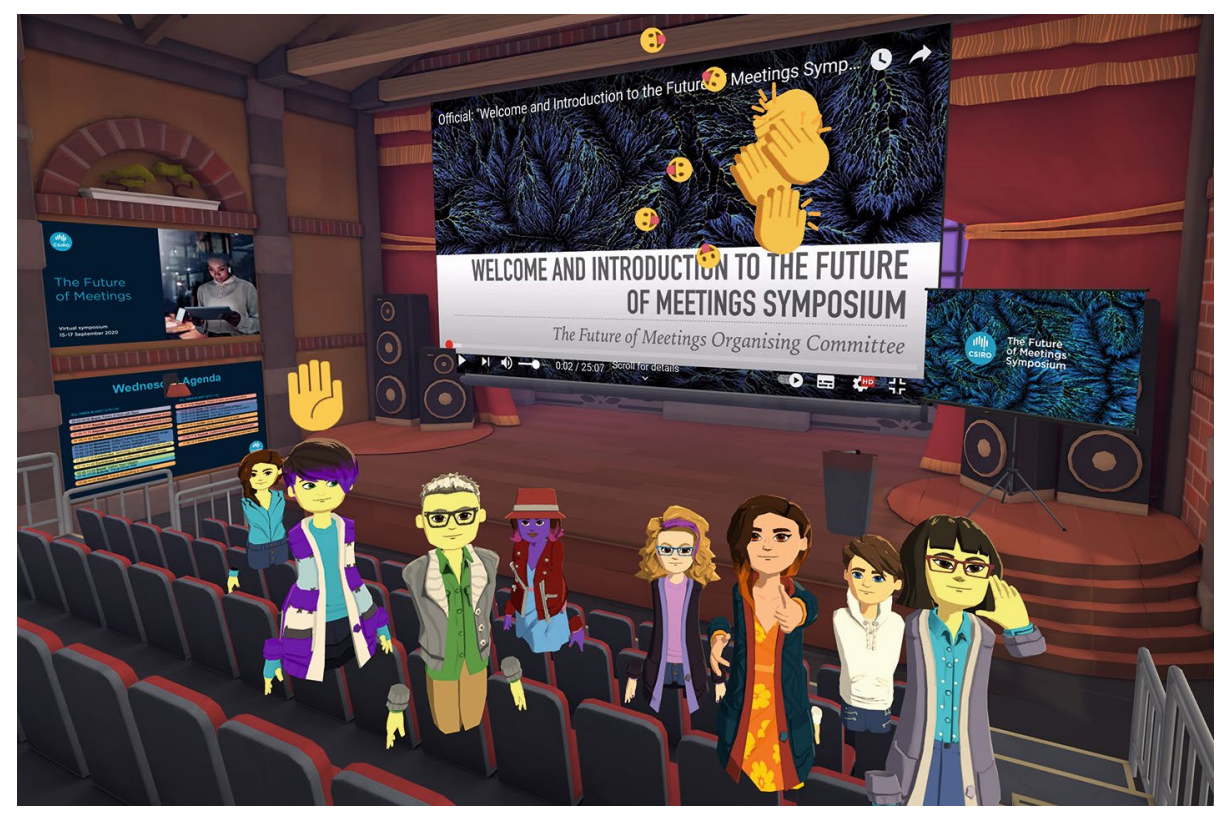

Fig. 1 | TFOM Organising Committee photo in AltspaceVR. Members of The Future of Meetings Organising Committee gathering in the virtual TFOM space, hosted in the social virtual reality platform AltspaceVR.

Commonwealth Scientific and Industrial

Research Organisation (CSIRO, Australia's national science agency) that took place in an entirely virtual venue. Our approach, from conception of TFOM to the collation of recommendations, was founded on experimentation and evaluation, with the aim to share a current perspective on what best practice might look like for future meetings, especially conferences. In planning we identified four key themes that we felt were critical to bring focus to: accessibility, inclusivity, sustainability and technology. We recently shared our experience and lessons learned from TFOM through a publicly available report ${ }^{4}$. The report provides extensive coverage of our process, outcomes and recommendations, and forms a comprehensive reference for those organizing conferences or interested in this topic.
For all the challenges faced in 2020, the disruptive nature of lockdown rapidly accelerated many trends that had been predicted and visible for years ${ }^{5,6}$. We are now at a critical turning point where we have the opportunity to benefit from these experiences and define what we want the future of scientific collaboration to be, but only if we actively choose to resist a return to 'normal' and strive for 'better' instead. We consolidated our core learnings from TFOM in a short list that can be abbreviated to DAISERVE: Digital-first, Accessibility, Inclusivity, Sustainability, Experimentation, Right tool/approach, Value and Evaluation. If these aspects are considered when designing interactions including but not limited to conferences, then this will facilitate our proposed template of current best practice. 


\section{Benefits and challenges of the virtual format}

There are many benefits that arise when adopting a virtual format instead of the in-person equivalent. In-person conferences typically have many barriers that create disadvantages in terms of accessibility. Access to funding, personal commitments, caring duties, health, ability to freely travel, mobility, disability, sustainability concerns - these factors among others have meant that our academic conferences have always been, often to a significant extent, inaccessible. We have had centuries to 'perfect' the art of meeting in person, and arguably such meetings are far from perfect. Perhaps the greatest advantage of a virtual format is the 'levelling of the playing field' across these many barriers to in-person, such that it is possible to create far more accessible and inclusive virtual forums. Critically, virtual meetings enable us to do significantly less harm to the environment as the need to reduce climate impact at all scales increases drastically ${ }^{7-10}$; we calculated that TFOM produced $1,420 \mathrm{~kg}$ of $\mathrm{CO}_{2}$ equivalent (which we offset in entirety via planting trees), while the in-person equivalent would have produced upwards of $280,000 \mathrm{~kg}$ of $\mathrm{CO}_{2}$ equivalent ${ }^{4}$. It also costs much less to both organize and attend virtual events and enables communication more broadly than ever before.

Another key advantage of the virtual format is the potential persistence and legacy of content. With virtual meetings, as well as standard content preservation, we can readily save discussions and have digitally delivered talks instantly available for viewing by those in different time zones or those who wish to engage with the content asynchronously. A digital format enables rapidly improving accessibility tools like automatic captioning and transcribing to be incorporated, increasing ease of participation. Digital collaborative tools like virtual whiteboards facilitate the reproduction and capture of brainstorming efforts, sometimes more effectively and equitably than in-person. Online archives can be produced from conferences and collaborations that, if supplemented by ways to connect and communicate, can result in conversations that stretch well beyond the boundaries of any given meeting or conference. An additional and serious consideration is the safety and confidence of junior researchers given the incidence of inappropriate interactions and sexual harassment at academic conferences ${ }^{11-13}$, in that virtual should allow them to connect safely from an environment that they are comfortable with.

Virtual environments, as we all know, do not come without challenges. The issue of attention span has been raised repeatedly due to many conferences porting their in-person programme directly to an online format, sometimes including a large number of parallel sessions. Most now acknowledge that the expectation of dialling into a video call for several hours is unacceptable, and yet this has been our standard in-person conference approach. There can be a coldness to the virtual format; it is sometimes hard to feel the same sense of community as when everyone is in a room together, able to make direct eye contact and detect subtle body language. Social bonds and networking have been harder to realize in a virtual space, which has particular importance for the junior members of the academic community since a personal interaction may affect their future career. Extroverts can be especially affected by the lack of in-person contact, though introverts may prefer the virtual format. Time zones present an undeniable obstacle in terms of bringing an international community together. Presence at a virtual meeting or conference often assumes that attendees have reliable access to suitable technologies and internet connections, which can be particularly challenging for those connecting from remote or developing areas.

These challenges are all real, but not insurmountable, nor do they outweigh the benefits gained by adopting a virtual format. It requires us to give deep thought to the concept of why we want or need to meet at all, and to disconnect from two key assumptions: firstly, that what we would do in-person is what we should do virtually, and secondly, that our standards for in-person were ever best practice to begin with. Live group attendance should be restricted to situations where it is most effective or necessary, and activities that can be done in a collaborative offline setting can supplement the need for lengthy online sessions. Breaks between content or meetings should be frequent, and in the case of conferences, should alternate between encouraging virtual social connection and giving attendees necessary downtime away from screens. Virtual community building takes effort, similar to building an in-person community, and this requires effort, invested by organizers, to encourage conversation and connection between attendees if this is an important goal, with particular awareness of how to maximize the benefits of such sessions for junior or less-connected members of a given community. Various technologies already exist that can take us beyond the standard 'Brady Bunch' video call format, including spatial audio/visual platforms, desktop-based virtual environments and immersive virtual/augmented reality platforms. Any given technology tool might work for one context but not another, which is why it is important to experiment and consider carefully the end goal of any interaction in choosing the appropriate solution. It is also possible to find ways to have shared meals/experiences/team building online, though these kinds of virtual social activities are really still in their infancy. For a comprehensive list of possible digital tools that may meet the needs described above, we refer readers to the 'Resources' section (XI) of the TFOM report ${ }^{4}$.

While the reality of a round planet will never go away, overcoming the challenge of time zones essentially depends on what absolutely needs to take place live and determining what can be done using collaborative online discussion tools. Finally, if technology access of attendees is a consideration, the platforms used for virtual meetings should not rely on live high-bandwidth connections. Generally, opting for platforms that adapt according to internet connections and operating systems is preferable. Making video content available in a recorded form before or immediately after an event can also be a helpful solution for low-bandwidth connections, additionally benefiting others for whom live attendance was not possible or preferable.

\section{What does the future look like?}

We propose that the primary goal of future conference organization should be that attendees have equivalent agency regardless of location. Coming out of 2020, we have seen many claims that hybrid will be the way of future meetings. We contend this is only viable and fair if these meetings are designed to be digital-first. By digital-first, we mean prioritizing the digital means of communication above all other ways of connecting even if there is an in-person component to the event. We first consider why the current hybrid model is fundamentally flawed compared to a digital-first approach.

The most common conception of a hybrid event is in-person with a remote connection. In the case of conferences and meetings, this typically manifests as a focus on the in-person event with the ability for speakers or attendees to connect to a virtual stream and these remote attendees are often invisible to physical participants. This approach does offer some advantages, namely allowing the richness of in-person interaction for those physically present, enabling broader access beyond the physical room and lowering environmental impact to some degree. However, there are significant drawbacks to this approach that 
limit its effectiveness and make it hard to recommend as best practice. The addition of a virtual option is usually considered a backup solution for those who cannot attend in person (which may be for personal reasons or disabilities), which often cements inequity and creates two classes of attendee. The environmental impact of hybrid remains high compared to purely digital (since people who can travel typically will). Additionally, this form of hybrid only has a chance of succeeding if the balance between in-person and virtual attendees is tipped in favour of the virtual. It is also not widely acknowledged that a hybrid event essentially doubles the workload for organizers, and in the case of hubs, requires geographically distributed yet well-synchronized organizational effort.

Can we design a better form of hybrid? Groups around the world have investigated and defined different models (see, for example, refs. ${ }^{14,15}$ ), while many contemporary conferences and meetings are empirically exploring the hybrid parameter space. Conversely, Trello and its now-parent company Atlassian have adopted a policy of 'all in the room or all virtual' to prevent inequalities in attendance ${ }^{16}$. In fact, many of these discussions and considerations can be traced back to early research into digital collaboration, where it was concluded that much of our problem with using digital means to reproduce face-to-face communication is the assumption that face-to-face is all we really need from virtual communication ${ }^{17}$. Ultimately, we advocate that digital-first mechanisms will be the binding element that holds future interactions together. Hybrid may then be a viable solution, if implemented by adopting a digital-first mindset in the design and execution of collaborative interactions.

\section{The importance of architecting digital events}

In terms of our role in organizing TFOM, we consciously identified, discussed and optimized every element of the symposium, which required considerable but worthwhile effort. In post-TFOM discussions, the related concept of a 'digital-meeting architect' was suggested as a role that may be needed in the future to ensure maximum success for virtual interactions of various kinds. This would be an organizational role, with focus on design and execution of the virtual nature of an event, and would help to address circumstances where virtual events fall short of expectation not due to intrinsic challenges but because they are missing the appropriate level of oversight and coordination by organizers.
Thinking of virtual interactions in the sense of an architecture captures some of the notion of actively and carefully constructing an effective event. Goal-oriented design and implementation of a virtual event takes effort, and while it will almost certainly be much cheaper than the in-person equivalent, it is by no means less work for organizers. It also highlights that we should be thinking of our virtual venues with the same attention to detail and desire for aesthetics as our physical venues. They should be aesthetically pleasing places to exist within, not simply focused on practicality or technical performance. While already relevant in terms of digital tools and media in common use, this aspect will soon have even more relevance as augmented and virtual reality technologies become increasingly prevalent in the workplace.

In the end, a successful virtual interaction is not dependent on any one factor, but on the sum of many parts that should be defined based on context and goals. While aspects of the interaction may be effectively outsourced (for example, video streaming management, recorded video editing and website design), there remains a necessity for everything to be coordinated carefully based on an overarching vision. For large events, this coordination has traditionally been provided by event management companies in close consultation with organizers, and there are trends towards this as a viable path for larger virtual interactions as event companies pivot to support digital more effectively. However, at this point in time we are finding the majority of conference organization companies do not yet have extensive 'digital architecture' expertise, which can result in large investment for suboptimal outcomes.

In our experience we found that virtual settings can be a great leveller in terms of required resources, and we believe that organizers themselves can (and should) take more direct ownership of events and conferences, likely reducing the required budget considerably for even large-scale events and meetings. Regardless of exactly who takes responsibility, a successful virtual event requires approaching the construction of the interaction with the right mindset. Thus, our key message on this topic of digital meeting architecture is that virtual can work extremely well, but only if you plan ahead and build the event appropriately for the virtual context.

\section{Recommendations and next steps} The intense period of disruption brought in by the global pandemic has given us an opportunity to reassess and redefine the ways in which we collaborate. As we contemplate a transition out of this disruptive era, it will be critical to avoid the temptation to dismiss 2020 as the sum of its worst parts without seeing the good that came out of it. While we are still exploring how best to be digital natives, we have seen tremendous progress in virtual communication and collaboration over the last few decades. To have come this far at remote collaboration in such a short time is incredible, and hints at some of the amazing things yet to come.

When we concluded TFOM, we urged our attendees to see this challenging period as one of learning, so that we can build the kind of future we want to be part of. Our collective experience in 2020 showed us the flaws in our approach to scientific collaboration, and bound us to innovative, more effective, accessible and sustainable ways of connecting. The questions to ask ourselves now, at the boundary between the present and the future, are what should we be doing next, and what changes can we make to build on the current situation for a better 'normal'? Should we return to face-to-face meetings and try to recover what we feel was lost during this disruptive period, without acknowledging the many benefits that we have gained from meeting virtually? Or is it worth forging ahead, along new pathways, with the recognition it will lead to discovering (sometimes the hard way) how to be better?

In the spirit of Tomfoolery's Great Realisation: "And so when we found the cure, and were allowed to go outside, we all preferred the world we found to the one we'd left behind."

Now is our chance to choose the accessible, the inclusive, the sustainable, the 'new normal' - not because it will be easy, but because it will lead to a better future.

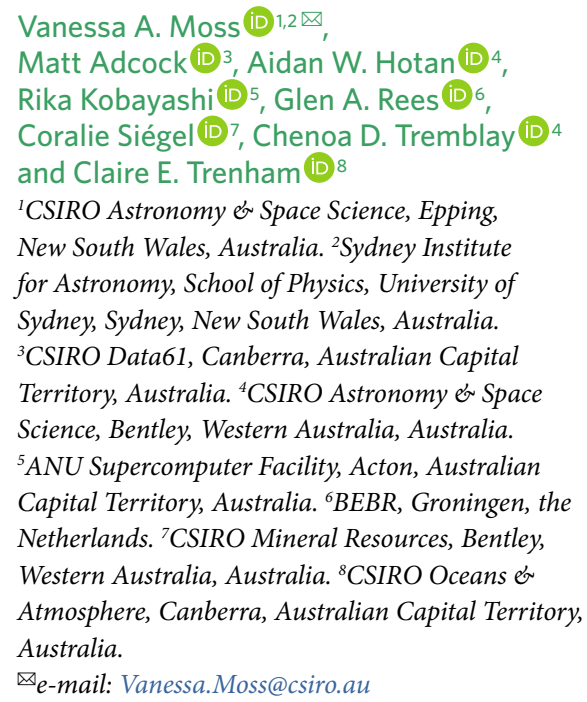


Published online: 16 March 2021

https://doi.org/10.1038/s41550-021-01325-z

References

1. Nat. Astron. 4, 1113 (2020).

2. Reimagining the Employee Experience (Fujitsu and Forrester, 2021); https://reprints2.forrester.com/\#/assets/2/1325 RES161255/report

3. McKie, R. Adapting to the new normal: hybrid working 2021. Pitcher Partners https://www.pitcher com.au/adapting-to-the-new-normal-hybrid-working2021 (2021).

4. Moss, V. A. et al. The Future of Meetings: Outcomes and Recommendations (Zenodo, 2020); https://zenodo.org/ record/4345562\#.X9wYmOkzbUJ
5. Parakala, K. Ten emerging trends shaping our new future. GHD https://www.ghd.com/en/perspectives ten-emerging-trends-shaping-our-new-future.aspx (2021).

6. Top 10 Emerging Technologies 2019 (World Economic Forum, 2019); http://www3.weforum.org/docs/WEF_Top_10_Emerging Technologies_2019_Report.pdf

7. Stevens, A. R. H. et al. Nat. Astron. 4, 843-851 (2020).

8. Burtscher, L. et al. Nat. Astron. 4, 823-825 (2020)

9. Glausiusz, J. Nature 589, 155-157 (2021).

10. Mega, E. R. Nature https://www.nature.com/articles/d41586-02003000-1 (2020).

11. Flores, N. M. Gend. Educ. 32, 137-144 (2020).

12. National Academies of Sciences, Engineering, and Medicine Sexual Harassment of Women: Climate, Culture, and Consequences in Academic Sciences, Engineering, and Medicine (eds Johnson, P. A. et al.) (National Academies Press, 2018).
13. Aycock, L. M. et al. Phys. Rev. Phys. Educ. Res. 15, 010121 (2019). 14. Fraser, H., Soanes, K., Jones, S. A., Jones, C. S. \& Malishev, M. Conserv. Biol. 31, 540-546 (2017).

15. Abbott, A. Nature 577, 13 (2020).

16. How to embrace remote work. Trello https://trello.com/enAU/remote-work-guide (2021).

17. Hollan, J. \& Stornetta, S. Beyond being there. In Proc. SIGCHI Conference on Human Factors in Computing Systems (eds Bauersfeld, P. et al.) 119-125 (ACM, 1992).

Acknowledgements

We thank P. Edwards, R. Ekers and E. Kerrison for feedback on this Comment that helped to improve its content.

Competing interests

The authors declare no competing interests. 